\title{
Fermion zero modes in the vortex background of a Chern-Simons-Higgs theory with a hidden sector
}

\author{
Gustavo Lozano, ${ }^{a}$ Azadeh Mohammadi $^{b}$ and Fidel A. Schaposnik ${ }^{c}$ \\ ${ }^{a}$ Departamento de Física, FCEYN Universidad de Buenos Aires \& IFIBA CONICET, \\ Pabellón 1 Ciudad Universitaria, 1428 Buenos Aires, Argentina \\ ${ }^{b}$ Departamento de Física, Universidade Federal da Paraíba, \\ 58.059-970, Caixa Postal 5.008, João Pessoa, PB, Brazil \\ ${ }^{c}$ Departamento de Física, Universidad Nacional de La Plata/IFLP/CICBA, \\ CC 67, 1900 La Plata, Argentina \\ E-mail: lozano@df.uba.ar, a.mohammadi@fisica.ufpb.br, \\ fidel@fisica.unlp.edu.ar
}

ABSTRACT: In this paper we study a $2+1$ dimensional system in which fermions are coupled to the self-dual topological vortex in $\mathrm{U}(1) \times \mathrm{U}(1)$ Chern-Simons theory, where both $\mathrm{U}(1)$ gauge symmetries are spontaneously broken. We consider two Abelian Higgs scalars with visible and hidden sectors coupled to a fermionic field through three interaction Lagrangians, where one of them violates the fermion number. Using a fine tuning procedure, we could obtain the number of the fermionic zero modes which is equal to the absolute value of the sum of the vortex numbers in the visible and hidden sectors.

KEYWORDS: Supersymmetric gauge theory, Solitons Monopoles and Instantons, ChernSimons Theories

ARXIV EPRINT: 1509.05925 


\section{Contents}

1 Introduction 1

2 The gauge field/Higgs background $\quad 2$

3 The Dirac zero-mode equation 3

$\begin{array}{lll}4 & \text { Summary and discussion } & 8\end{array}$

\section{Introduction}

Zero-eigenvalue modes in the Dirac equation for fermions coupled to a topologically nontrivial background can have remarkable physical consequences in systems belonging to a large domain in physics, going from high energy to condensed matter physics. A typical example in the former case is the so-called QCD $\mathrm{U}(1)_{A}$ problem which can be explained taking into account the zero modes of Dirac fermions in an instanton background [1]. Also in many condensed matter systems, as for example in superconducting graphene or in topological insulators, zero-mode fermions in a vortex background could play a central role (see [2] and references therein).

Zero modes in 2+1-dimensional Dirac equations for fermions minimally coupled to an Abelian Higgs model vortex [3]-[4] were first studied by Nohl [5] and de Vega [6]. Jackiw and Rossi [7] reconsidered this problem adding a fermion number violating interaction between fermions and the Higgs field. They set the fermion mass term to zero but nevertheless a mass was generated dynamically via the fermion-Higgs interaction. They found that the resulting Dirac equation has $|n|$ zero-mode solutions in the $n$-vortex background field.

When a Chern-Simons term is included in the $2+1$-dimensonal Lagrangian, vortex solutions are electrically charged [8]-[13]. In this case the search for Dirac equation zero modes is considerably more involved due to the existence of an electrostatic potential. Grignani and Nardelli [14] studied in detail the case in which the vortex background corresponds to a Chern-Simons-Higgs action [11]-[12] including cases with Yukawa-like interactions between fermions and the Higgs field. Also, by taking the vortex background as the one arising from a $\mathcal{N}=2$ supersymmetric Chem-Simons-Higgs model, Lee et al. found an interesting connection between fermionic and bosonic zero modes [15].

Recently, the study of gauge theories in which a hidden sector is coupled to the Standard Model or its supersymmetric extensions has received much attention in connection with dark matter, supersymmetry breaking and phenomenological superstring studies (see [16]-[17] and references therein). In this context self-dual vortex solutions in the bosonic sector of $\mathcal{N}=2, \mathrm{U}(1) \times \mathrm{U}(1)$ planar gauge theories coupled to Higgs scalars and including Chern-Simons terms have been considered in [18]. There have been also results 
concerning the case of a Maxwell-Chern-Simons-Higgs model with vortex solutions which might have interesting implications in condensed matter problems [19-21].

It is the purpose of the present paper to study the zero-mode Dirac equation for a fermion in the background of the self-dual vortex solutions referred above, namely a $\mathrm{U}(1) \times \mathrm{U}(1)$ Chern-Simons gauge theory, each sector coupled charged scalars with a mixing between both sectors given in [18]. We first consider the case in which both $\mathrm{U}(1)$ gauge symmetries are spontaneously broken so that two distinct topological Chern-Pontryagin numbers characterize the vortex magnetic fluxes and electric charges. We also comment on the case in which one of the Higgs scalars is absent (no symmetry breaking in the corresponding sector) so that there is just one topological number but still magnetic flux and electric charge confined in a flux tube.

\section{The gauge field/Higgs background}

The $2+1$ dimensional bosonic sector of the $\mathcal{N}=2$ supersymmetric $\mathrm{U}(1) \times U_{h}(1)$ ChernSimons gauge theory coupled to Higgs scalars analyzed in [18] reads

$$
L=\kappa \epsilon^{\mu \nu \rho} A_{\mu} \partial_{\nu} A_{\rho}+\kappa_{h} \epsilon^{\mu \nu \rho} C_{\mu} \partial_{\nu} C_{\rho}+2 \xi \epsilon^{\mu \nu \rho} A_{\mu} \partial_{\nu} C_{\rho}+\left|D_{\mu}[A] \phi\right|^{2}+\left|D_{\mu}[C] \eta\right|^{2}-V_{\text {eff }}[\phi, \eta] .
$$

Here

$$
D_{\mu}^{A} \phi=\partial_{\mu} \phi-i q A_{\mu} \phi, \quad D_{\mu}^{C} \eta=\partial_{\mu} \eta-i q_{h} C_{\mu} \eta
$$

where $q$ is the charge coupling the $\mathrm{U}(1)$ gauge $A_{\mu}$ to the Higgs scalar $\phi$ while $q_{h}$ plays the same role for the $U_{h}(1)$ gauge field $G_{\mu}$ and the scalar $\eta$. Concerning $V_{\text {eff }}[\phi, \eta]$, supersymmetry forces the sixth order potential to take the form

$$
\begin{aligned}
V_{\mathrm{eff}}= & \frac{1}{4\left(\kappa \kappa_{h}-\xi^{2}\right)}\left(\left(\kappa_{h} q\left(|\phi|^{2}-\phi_{0}^{2}\right)+\xi q_{h}\left(|\eta|^{2}-\eta_{0}^{2}\right)\right)^{2} e^{2}|\phi|^{2}\right. \\
& \left.+\left(\kappa q_{h}\left(|\eta|^{2}-\eta_{0}^{2}\right)+\xi q\left(|\phi|^{2}-\phi_{0}^{2}\right)\right)^{2} g^{2}|\eta|^{2}\right)
\end{aligned}
$$

where $\xi^{2}<\kappa \kappa_{h}$. Note that the Lagrangian includes a gauge mixing term (with parameter $\xi)$ which also implies a Higgs portal mixing the two scalars in the symmetry breaking potential.

The vortex configurations ansatz takes the following form

$$
\begin{array}{lll}
\phi=\phi_{0} f(r) e^{i n \theta}, & A_{\varphi}=-\frac{A(r)}{q r}, & A_{r}=0 \\
\eta=\eta_{0} h(r) e^{i k \theta}, & C_{\varphi}=-\frac{C(r)}{q_{h} r}, & C_{r}=0
\end{array}
$$

where $n$ and $k$ are the winding numbers associated to each Higgs scalar.

The Bogomolny equations for static configurations associated to Lagrangian (2.1) can be easily obtained from vanishing of the SUSY charges acting on physical states of the 
extended supersymmetric model [18]. They read

$$
\begin{aligned}
D_{1}^{A} \phi & =\operatorname{sgn}(n) i D_{2}^{A} \phi \\
D_{1}^{C} \eta & =\operatorname{sgn}(k) i D_{2}^{C} \eta \\
B & =-\operatorname{sgn}(n) \frac{q^{2}|\phi|^{2}}{2\left(\kappa \kappa_{h}-\xi^{2}\right)(\kappa-\xi)}\left(q \kappa_{h}\left(|\phi|^{2}-\phi_{0}^{2}\right)+\xi q_{h}\left(|\eta|^{2}-\eta_{0}^{2}\right)\right), \\
B_{h} & =-\operatorname{sgn}(k) \frac{q_{h}^{2}|\eta|^{2}}{2\left(\kappa \kappa_{h}-\xi^{2}\right)\left(\kappa_{h}-\xi\right)}\left(q_{h} \kappa\left(|\eta|^{2}-\eta_{0}^{2}\right)+\xi q\left(|\phi|^{2}-\phi_{0}^{2}\right)\right), \\
A_{0} & =-\operatorname{sgn}(n) \frac{1}{\left(\kappa \kappa_{h}-\xi^{2}\right)}\left(\kappa_{h} q\left(|\phi|^{2}-\phi_{0}^{2}\right)+\xi q_{h}\left(|\eta|^{2}-\eta_{0}^{2}\right)\right), \\
C_{0} & =-\operatorname{sgn}(k) \frac{1}{\left(\kappa \kappa_{h}-\xi^{2}\right)}\left(\kappa q_{h}\left(|\eta|^{2}-\eta_{0}^{2}\right)+\xi q\left(|\phi|^{2}-\phi_{0}^{2}\right)\right),
\end{aligned}
$$

where the magnetic fields are defined as $B=F_{12}[A]$ and $B_{h}=F_{12}[G]$ and $\operatorname{sgn}(n)=\frac{|n|}{n}$. The numerical solutions, which also solve the second order field equations are described in [18].

\section{The Dirac zero-mode equation}

Extending the proposal in [14] for the ordinary Chern-Simons-Higgs self-dual model, we shall consider the following Dirac Lagrangian for the case in which a hidden sector is included

$$
L_{D}=\bar{\psi}\left(\gamma^{\mu}\left(i \partial_{\mu}-e A_{\mu}-e_{h} C_{\mu}\right)-m\right) \psi+L_{\phi \eta}+L_{\phi}+L \eta
$$

with

$$
\begin{aligned}
L_{\phi \eta} & =-\frac{i}{2} g \phi \eta \bar{\psi} \psi^{c}+\frac{i}{2} g^{*} \phi^{*} \eta^{*} \bar{\psi}^{c} \psi, \\
L_{\phi} & =-g_{\phi}|\phi|^{2} \bar{\psi} \psi \\
L_{\eta} & =-g_{\eta}|\eta|^{2} \bar{\psi} \psi .
\end{aligned}
$$

We are working in $2+1$ dimensions and hence the lowest dimension representation for Dirac $\gamma$-matrices is two. They can be chosen as

$$
\gamma_{0}=\sigma^{3}, \quad \gamma_{1}=i \sigma^{2}, \quad \gamma_{2}=-i \sigma^{1}
$$

where $\sigma_{i}$ are the Pauli matrices. This leads to $\alpha$-Dirac matrices of the form $\vec{\alpha}=\left(\sigma^{1}, \sigma^{2}\right)$ and $\beta=\sigma^{3}$. Concerning the charge conjugated spinor $\psi^{c}$, it is related to $\bar{\psi}$ according to

$$
\psi^{c \alpha}=C^{\alpha \beta} \bar{\psi}^{\beta}
$$

with $C^{\alpha \beta}$ the charge conjugation matrix.

All three interaction Lagrangians, eqs. (3.2)-(3.4), correspond to power counting renormalizable intersections in $2+1$ dimensions. It should be also noted that similar fermion/scalar interactions arise in the supersymmetric extensions of Abelian Higgs models [22]. Also note that in the presence of interaction (3.2), fermion number which corresponds to an invariance under the global transformation $\psi \rightarrow \exp (i \alpha) \psi$ is violated. 
In view of the covariant derivatives definitions given in (2.2), gauge invariance implies that gauge and matter fields should change according to

$$
\begin{aligned}
A_{\mu} \stackrel{\Lambda}{\longrightarrow} A_{\mu}^{\prime} & =A_{\mu}-\partial_{\mu} \Lambda(x) & C_{\mu} \stackrel{\Lambda_{h}}{\longrightarrow} C_{\mu}^{\prime} & =C_{\mu}-\partial_{\mu} \Lambda_{h}(x) \\
\phi \stackrel{\Lambda}{\longrightarrow} \phi^{\prime} & =\exp (i q \Lambda(x)) \phi & \eta \stackrel{\Lambda_{h}}{\longrightarrow} \eta^{\prime} & =\exp \left(i q_{h} \Lambda_{h}(x)\right) \eta \\
\psi \stackrel{\Lambda}{\longrightarrow} \psi^{\prime} & =\exp (i e \Lambda(x)) \psi & \psi \stackrel{\Lambda_{h}}{\longrightarrow} \psi^{\prime} & =\exp \left(i e_{h} \Lambda_{h}(x)\right) \phi
\end{aligned}
$$

In the presence of the fermion number violating interaction Lagrangian (3.2), the mattergauge fields couplings should obey the following relations in order to have a $\mathrm{U}(1) \times \mathrm{U}(1)_{h}$ gauge invariant theory [7]

$$
q=2 e, \quad q_{h}=2 e_{h}
$$

Interaction Lagrangians $L_{\phi}$ and $L_{\eta}$ do not impose any relation between charges.

Notice that in the present case, like in [14], the fermion-scalar interaction is quadratic in the scalar field and the coupling constant is dimensionless, which contrasts with [7] where the coupling is lineal in the scalar. On the other hand, like in [7], the fermion number violating term involves the two scalar fields and consequently the scalar charge is twice the electron charge, unlike the case in [14] where the scalar charge is the same as the fermion charge.

The Dirac equation following Lagrangian (3.1) takes the form

$$
\begin{aligned}
& \left(i \partial_{0}-e A_{0}(\vec{x})-e_{h} C_{0}(\vec{x})\right) \psi(\vec{x}, t)=\left(-\vec{\alpha} \cdot\left(i \vec{\nabla}+e \vec{A}+e_{h} \vec{C}\right)+\beta m\right) \psi(\vec{x}, t) \\
& -g f(r) \phi_{0} \eta_{0} h(r) \exp (i(n+k) \varphi) \sigma^{2} \psi^{*}(t, \vec{x})+\left(g_{\phi} \phi_{0}^{2} f^{2}(r)+g_{\eta} \eta_{0}^{2} h^{2}(r)\right) \sigma^{3} \psi(t, \vec{x})=0 .
\end{aligned}
$$

Following [7]-[14] we make the two phases ansatz in order to factor out time dependence

$$
\psi(t, r, \varphi)=\exp (-i E t) \Psi^{+}(r, \varphi)+\exp (i E t) \Psi^{-}(r, \varphi) .
$$

Now, for the zero-mode solutions the two equations collapse to one which takes the form

$$
\begin{aligned}
& \left(-\vec{\alpha} \cdot\left(i \vec{\nabla}+e \vec{A}+e_{h} \vec{C}\right)+e A_{0}+e_{h} C_{0}+\beta m\right) \Psi(r, \varphi) \\
& -g f(r) \phi_{0} \eta_{0} h(r) \exp (i(n+k) \varphi) \sigma^{2} \Psi^{*}(r, \varphi)+\left(g_{\phi} \phi_{0}^{2} f^{2}(r)+g_{\eta} \eta_{0}^{2} h^{2}(r)\right) \sigma^{3} \Psi(r, \varphi)=0 .
\end{aligned}
$$

In terms of upper and lower components of the spinor field $\Psi, \Psi=\left(\begin{array}{l}\Psi_{U} \\ \Psi_{L}\end{array}\right)$, the above equation can be written in the form of the coupled system,

$$
\begin{aligned}
& \left(e A_{0}+e_{h} C_{0}+m\right) \Psi_{U}-i e^{-i \varphi}\left(\partial_{r}-\frac{i}{r} \partial_{\varphi}+\frac{1}{2}(A(r)+C(r))\right) \Psi_{L} \\
& +i g f(r) \phi_{0} \eta_{0} h(r) \exp (i(n+k) \varphi) \Psi_{L}^{*}(r, \varphi)+\left(g_{\phi} \phi_{0}^{2} f^{2}(r)+g_{\eta} \eta_{0}^{2} h^{2}(r)\right) \Psi_{\mathrm{U}}(r, \varphi)=0,
\end{aligned}
$$

and

$$
\begin{aligned}
& \left(e A_{0}+e_{h} C_{0}-m\right) \Psi_{L}-i e^{i \varphi}\left(\partial_{r}+\frac{i}{r} \partial_{\varphi}-\frac{1}{2}(A(r)+C(r))\right) \Psi_{U} \\
& -i g f(r) \phi_{0} \eta_{0} h(r) \exp (i(n+k) \varphi) \Psi_{U}^{*}(r, \varphi)-\left(g_{\phi} \phi_{0}^{2} f^{2}(r)+g_{\eta} \eta_{0}^{2} h^{2}(r)\right) \Psi_{L}(r, \varphi)=0 .
\end{aligned}
$$


Proposing the two-phase ansatz as

$$
\begin{aligned}
& \Psi_{U}=U_{U} e^{i m \varphi}+V_{U} e^{i(n+k-m-1) \varphi}, \\
& \Psi_{L}=U_{L} e^{i(m+1) \varphi}+V_{L} e^{i(n+k-m) \varphi},
\end{aligned}
$$

with $m \in \mathbf{Z}$, we obtain the following set of coupled equations

$$
\begin{gathered}
\left(e A_{0}+e_{h} C_{0}+m\right) U_{U}-i\left[\partial_{r}+\frac{m+1}{r}+\frac{1}{2}(A(r)+C(r))\right] U_{L} \\
+i g f(r) \phi_{0} \eta_{0} h(r) V_{L}^{*}+\left(g_{\phi} \phi_{0}^{2} f^{2}(r)+g_{h} \eta_{0}^{2} h^{2}(r)\right) U_{U}=0, \\
\left(e A_{0}+e_{h} C_{0}+m\right) V_{U}-i\left[\partial_{r}+\frac{n+k-m}{r}+\frac{1}{2}(A(r)+C(r))\right] V_{L} \\
+i g f(r) \phi_{0} \eta_{0} h(r) U_{L}^{*}+\left(g_{\phi} \phi_{0}^{2} f^{2}(r)+g_{h} \eta_{0}^{2} h^{2}(r)\right) V_{U}=0, \\
\left(e A_{0}+e_{h} C_{0}-m\right) U_{L}-i\left[\partial_{r}-\frac{m}{r}-\frac{1}{2}(A(r)+C(r))\right] U_{U} \\
-i g f(r) \phi_{0} \eta_{0} h(r) V_{U}^{*}-\left(g_{\phi} \phi_{0}^{2} f^{2}(r)+g_{h} \eta_{0}^{2} h^{2}(r)\right) U_{L}=0, \\
\left(e A_{0}+e_{h} C_{0}-m\right) V_{L}-i\left[\partial_{r}-\frac{n+k-m-1}{r}-\frac{1}{2}(A(r)+C(r))\right] V_{U} \\
-i g f(r) \phi_{0} \eta_{0} h(r) U_{U}^{*}-\left(g_{\phi} \phi_{0}^{2} f^{2}(r)+g_{h} \eta_{0}^{2} h^{2}(r)\right) V_{L}=0 .
\end{gathered}
$$

Defining the following relations

$$
\mathcal{M} \equiv \frac{q^{2} \phi_{0}^{2}}{|\kappa|}, \quad \mathcal{N} \equiv \frac{q_{h}^{2} \eta_{0}^{2}}{\left|\kappa_{h}\right|}
$$

one can rewrite the zeroth components of the gauge fields in eq. (2.6) in the form

$$
\begin{aligned}
e A_{0} & =\operatorname{sgn}(n)\left(\frac{|\kappa| \kappa_{h} e}{q\left(\kappa \kappa_{h}-\xi^{2}\right)} \mathcal{M}\left(1-f^{2}\right)+\frac{\left|\kappa_{h}\right| \xi e}{q_{h}\left(\kappa \kappa_{h}-\xi^{2}\right)} \mathcal{N}\left(1-h^{2}\right)\right), \\
e_{h} C_{0} & =\operatorname{sgn}(k)\left(\frac{\left|\kappa_{h}\right| \kappa e_{h}}{q_{h}\left(\kappa \kappa_{h}-\xi^{2}\right)} \mathcal{N}\left(1-h^{2}\right)+\frac{|\kappa| \xi e_{h}}{q\left(\kappa \kappa_{h}-\xi^{2}\right)} \mathcal{M}\left(1-f^{2}\right)\right) .
\end{aligned}
$$

As a result, we obtain

$$
e A_{0}+e_{h} C_{0}=\left(|\kappa| \frac{\operatorname{sgn}(n) \kappa_{h} e+\operatorname{sgn}(k) \xi e_{h}}{\left(\kappa \kappa_{h}-\xi^{2}\right)} \frac{\mathcal{M}}{q}\left(1-f^{2}\right)+\left|\kappa_{h}\right| \frac{\operatorname{sgn}(k) \kappa e_{h}+\operatorname{sgn}(n) \xi e}{\left(\kappa \kappa_{h}-\xi^{2}\right)} \frac{\mathcal{N}}{q_{h}}\left(1-h^{2}\right)\right)
$$

Now, calling

$$
\begin{gathered}
l \equiv \frac{\kappa \kappa_{h}-\xi^{2}}{\kappa\left[\operatorname{sgn}(n) \kappa_{h} e+\operatorname{sgn}(k) \xi e_{h}\right]} q, \\
l_{h} \equiv \frac{\kappa \kappa_{h}-\xi^{2}}{\kappa_{h}\left[\operatorname{sgn}(k) \kappa e_{h}+\operatorname{sgn}(n) \xi e\right]} q_{h},
\end{gathered}
$$

we finally get

$$
e A_{0}+e_{h} C_{0}=\left(\operatorname{sgn}(\kappa) \frac{\mathcal{M}}{l}\left(1-f^{2}\right)+\operatorname{sgn}\left(\kappa_{h}\right) \frac{\mathcal{N}}{l_{h}}\left(1-h^{2}\right)\right),
$$


which can be written as the extension of Grignani-Nardeli result to the $\mathrm{U}(1) \times \mathrm{U}(1)$ model if one defines

$$
e \hat{A}_{0} \equiv \operatorname{sgn}(\kappa) \frac{\mathcal{M}}{l}, \quad e_{h} \hat{C}_{0} \equiv \operatorname{sgn}\left(\kappa_{h}\right) \frac{\mathcal{N}}{l_{h}}
$$

It results in

$$
e A_{0}+e_{h} C_{0}=e \hat{A}_{0}\left(1-f^{2}\right)+e_{h} \hat{C}_{0}\left(1-h^{2}\right) .
$$

Let us define $I_{0}$ as

$$
I_{0} \equiv\left(\left(e \hat{A}_{0}-g_{\phi} \phi_{0}^{2}\right)\left(1-f^{2}\right)+\left(e_{h} \hat{C}_{0}-g_{\eta} \eta_{0}^{2}\right)\left(1-h^{2}\right)+M_{f}\right)
$$

with

$$
M_{F} \equiv m+g_{\phi} \phi_{0}^{2}+g_{\eta} \eta_{0}^{2} .
$$

Then, the terms containing an $U_{U}$ factor in eq. (3.15) and a $V_{U}$ factor in eq. (3.16) take the form $I_{0} U_{U}$ and $I_{0} V_{V}$, respectively. Analogously, the terms containing $U_{L}$ and $V_{L}$ in eqs. (3.17)-(3.18) are of the form $J_{0} U_{l}$ and $J_{0} V_{L}$ with

$$
J_{0} \equiv\left(\left(e \hat{A}_{0}+g_{\phi} \phi_{0}^{2}\right)\left(1-f^{2}\right)+\left(e_{h} \hat{C}_{0}+g_{h} \eta_{0}^{2}\right)\left(1-h^{2}\right)-M_{f}\right) .
$$

Then, in order to find non-trivial zero-mode solutions the analysis in [14] for the case of just one sector can be extended to the present model leading to the following fine tuning conditions

$$
\begin{aligned}
M_{f} & =0, \\
\hat{A}_{0} & = \pm \frac{g_{\phi}}{e} \phi_{0}^{2}, \\
\hat{C}_{0} & = \pm \frac{g_{h}}{e_{h}} \eta_{0}^{2},
\end{aligned}
$$

so that either $I_{0}=0$ or $J_{0}=0$, depending on the sign choice.

Let us first consider the case in which the signs in eq. (3.30) and in eq. (3.31) are negative. Under these conditions, the upper components, $U_{U}$ and $V_{U}$ completely decouple from the lower components, so that both of them can be taken equal to zero. Then eqs. (3.15)-(3.16) take the simple form

$$
\begin{aligned}
\left(\partial_{r}+\frac{m+1}{r}+\frac{1}{2}(A(r)+C(r))\right) U_{L} & =g f(r) \phi_{0} \eta_{0} h(r) V_{L}^{*}, \\
\left(\partial_{r}+\frac{n+k-m}{r}+\frac{1}{2}(A(r)+C(r))\right) V_{L} & =g f(r) \phi_{0} \eta_{0} h(r) U_{L}^{*} .
\end{aligned}
$$

These equations can be simplified by defining new functions $\tilde{U}_{L}$ and $\tilde{V}_{L}$ through the relations

$$
\begin{aligned}
& U_{L}=\exp \left(-\frac{1}{2} \int_{0}^{r} d r^{\prime}\left(A\left(r^{\prime}\right)+C\left(r^{\prime}\right)\right)\right) r^{-m-1} \tilde{U}_{L}, \\
& V_{L}=\exp \left(-\frac{1}{2} \int_{0}^{r} d r^{\prime}\left(A\left(r^{\prime}\right)+C\left(r^{\prime}\right)\right)\right) r^{m-(n+k)} \tilde{V}_{L},
\end{aligned}
$$


so that one now has

$$
\begin{aligned}
& \partial_{r} \tilde{U}_{L}=g \phi_{0} \eta_{0} f(r) h(r) \tilde{V}_{L}^{*} r^{(2 m-(k+n)+1)}, \\
& \partial_{r} \tilde{V}_{L}=g \phi_{0} \eta_{0} f(r) h(r) \tilde{U}_{L}^{*} r^{(-2 m+(k+n)-1)} .
\end{aligned}
$$

Because of the properties of the vortex solutions, the exponentials in eq. (3.33) go to 1 at the origin and to $r^{-(n+k) / 2}$ at infinity.

One can now obtain a set of real equations by writing

$$
\tilde{U}_{L}=\exp \left(i \omega_{L}\right) \hat{U}_{L}, \quad \tilde{V}_{L}=\exp \left(-i \omega_{L}\right) \hat{V}_{L}
$$

with $\omega_{L}$ an arbitrary phase and $\hat{U}_{L}$ and $\hat{V}_{L}$ solutions of the equations

$$
\begin{aligned}
& \partial_{r} \hat{U}_{L}=g \phi_{0} \eta_{0} f(r) h(r) \hat{V}_{L} r^{(2 m-(n+k)+1)}, \\
& \partial_{r} \hat{V}_{L}=g \phi_{0} \eta_{0} f(r) h(r) \hat{U}_{L} r^{(-2 m+(n+k)-1)} .
\end{aligned}
$$

From these equations we see that $\hat{U}_{L}$ and $\hat{V}_{L}$, apart from a power behavior, have an exponential behavior $\exp \left( \pm\left|g \phi_{0} \eta_{0}\right| r\right)$. In order to have regular zero-mode solutions, $U_{L}$ and $V_{L}$ should be well-behaved at the origin as well as at infinity. It follows that

$$
\begin{array}{cl}
U_{L} \underset{\text { small } \mathrm{r}}{\sim} r^{-m-1}, & r^{m-(n+k)+|n|+|k|+1} \\
V_{l} \underset{\text { small r }}{\sim} r^{m-(n+k)}, & r^{-m+|n|+|k|}
\end{array}
$$

All the solutions to this equations are regular provided the following inequalities hold

$$
(n+k) \leq m \leq-1,
$$

which in turn implies $n+k<0$ and therefore the number of zero modes is $|n+k|$.

If one chooses $A_{0}$ and $C_{0}$ with positive signs instead of those chosen in the analysis above, the analysis goes the same. One can take $U_{L}=V_{L}=0$ and instead of eq. (3.37) we have

$$
\begin{array}{ll}
U_{L} \underset{\text { small r }}{\sim} r^{m}, & r^{-m+(n+k)+|n|+|k|} \\
V_{l} \underset{\text { small r }}{\sim} r^{-m+(n+k)-1}, & r^{m+|n|+|k|+1}
\end{array}
$$

leading to the inequalities

$$
n+k-1 \geq m \geq 0,
$$

which can only be satisfied for positive $n+k$. Therefore, it results in the existence of $(n+k)$ zero modes.

The final form of the zero-energy eigenfunctions for the case $n+k<0$ is

$$
\begin{aligned}
\psi_{(n+k)<0}(\vec{r})= & \exp \left(-\frac{1}{2} \int_{0}^{r} d r^{\prime}\left(A\left(r^{\prime}\right)+C\left(r^{\prime}\right)\right)\right) \\
& \times\left(e^{i\left((m+1) \varphi+\omega_{L}\right)} r^{-m-1} \hat{U}_{L}+e^{i\left((n+k-m) \varphi-\omega_{L}\right)} r^{m-(k+n)} \hat{V}_{L}\right)\left(\begin{array}{l}
0 \\
1
\end{array}\right),
\end{aligned}
$$


while for $n+k>0$ one has

$$
\begin{aligned}
\psi_{(n+k)>0}(\vec{r})= & \exp \left(-\frac{1}{2} \int_{0}^{r} d r^{\prime}\left(A\left(r^{\prime}\right)+C\left(r^{\prime}\right)\right)\right) \\
& \times\left(e^{i\left(m \varphi+\omega_{U}\right)} r^{m} \hat{U}_{U}+e^{i\left((n+k-m-1) \varphi-\omega_{U}\right)} r^{(k+n)-m-1} \hat{V}_{U}\right)\left(\begin{array}{l}
1 \\
0
\end{array}\right) .
\end{aligned}
$$

One can see that as a result of fermion number violation, $\psi$ is an eigenstate of particle conjugation as defined in [7],

$$
\sigma^{3} \psi=\operatorname{sgn}(n+k) \psi
$$

One can also find zero modes in the absence of fermion-number violating terms. In this case $q / e$ and $q_{h} / e_{h}$ are not fixed by gauge invariance so that, as in refs. [5]-[6] for just a visible sector, the zero modes will depend on this ratio.

\section{Summary and discussion}

In this paper we have found all zero modes of fermions in the background of vortex solutions of a Chern-Simons-Higgs model with visible and hidden gauge and Higgs fields. Apart from their quantized magnetic flux, the vortices are electrically charged and they are solutions of self-dual equations found in [18] by considering the $\mathcal{N}=2$ supersymmetric extension of the model.

What we have analyzed in this paper is the Dirac equation for a single fermion field that couples to the vortex background fields of the two sectors. It could be of interest to instead consider the zero modes of the $\mathcal{N}=2$ supersymmetric model with fermions in the two sectors, a $\mathrm{U}(1) \times \mathrm{U}(1)$ extension of the analysis presented in [15] for just one sector, where a simple connection between all independent fermion and boson zero modes is established through a formula that converts every fermion zero mode into a correspondent bosonic zero mode. Analogous relation should exist when the two U(1) sectors are considered.

Another direction to explore has to do with possible condensed matter applications. In this respect, we have seen that zero-modes as given in eq. (3.42) can be interpreted as their own antiparticles and hence as discussed in [2] they can play a relevant role in describing certain quantum Hall states and some exotic superconductors. Also in connection with superconductivity it was shown in [18] that when one of the two U(1) symmetries remains unbroken (for example when no Higgs field in the corresponding sector is present) the magnetic and electric fields are proportional (and so are the magnetic flux and the electric charge). In this case the model makes contact with the one considered by Anber et al. [19-21], except that no Maxwell term is included in any of the two sectors. It could be interesting to see whether the condensed matter applications discussed in [19-21], in particular the possibility of superconductivity at any temperature, can be analyzed from the fermionic zero modes side. 


\section{Acknowledgments}

A.M. thanks Conselho Nacional de Desenvolvimento Científico e Tecnológico (CNPq) for the financial support within the frame of Grant No. 501795/2013-8. F.A.S. is associated to CICBA and financially supported by PIP-CONICET, PICT-ANPCyT, UNLP and CICBA grants. G.S.L is finacially supported by PIP-CONICET and UBA.

Open Access. This article is distributed under the terms of the Creative Commons Attribution License (CC-BY 4.0), which permits any use, distribution and reproduction in any medium, provided the original author(s) and source are credited.

\section{References}

[1] S. Coleman, The Uses of Instantons, in Aspects of Symmetries: Selected Erice lectures, Cambridge University Press, Cambdrige, U.K. (1985), pg. 265-350.

[2] F. Wilczek, Majorana returns, Nature Phys. 5 (2009) 614.

[3] A.A. Abrikosov, On the Magnetic properties of superconductors of the second group, Sov. Phys. JETP 5 (1957) 1174 [INSPIRE].

[4] H.B. Nielsen and P. Olesen, Vortex Line Models for Dual Strings, Nucl. Phys. B 61 (1973) 45 [InSPIRE].

[5] C.R. Nohl, Bound State Solutions of the Dirac Equation in Extended Hadron Models, Phys. Rev. D 12 (1975) 1840 [inSPIRE].

[6] H.J. de Vega, Fermions and Vortex Solutions in Abelian and Nonabelian Gauge Theories, Phys. Rev. D 18 (1978) 2932 [INSPIRE].

[7] R. Jackiw and P. Rossi, Zero Modes of the Vortex-Fermion System, Nucl. Phys. B 190 (1981) 681 [InSPIRE].

[8] S.K. Paul and A. Khare, Charged Vortices in Abelian Higgs Model with Chern-Simons Term, Phys. Lett. B 174 (1986) 420 [Erratum ibid. 177B (1986) 453] [INSPIRE].

[9] H.J. de Vega and F.A. Schaposnik, Electrically Charged Vortices in Nonabelian Gauge Theories With Chern-Simons Term, Phys. Rev. Lett. 56 (1986) 2564 [INSPIRE].

[10] H.J. de Vega and F.A. Schaposnik, Vortices and electrically charged vortices in nonAbelian gauge theories, Phys. Rev. D 34 (1986) 3206 [InSPIRE].

[11] J. Hong, Y. Kim and P.Y. Pac, On the Multivortex Solutions of the Abelian Chern-Simons-Higgs Theory, Phys. Rev. Lett. 64 (1990) 2230 [InSPIRE].

[12] R. Jackiw and E.J. Weinberg, Self-dual Chern-Simons vortices, Phys. Rev. Lett. 64 (1990) 2234 [INSPIRE].

[13] L.F. Cugliandolo and G. Lozano, Zero Modes of the Vortex Fermion System: The Nonabelian Case, Phys. Rev. D 39 (1989) 3093 [InSPIRE].

[14] G. Grignani and G. Nardelli, Threshold bound states and zero modes of fermions in a selfdual Chern-Simons vortex background, Phys. Rev. D 43 (1991) 1919 [INSPIRE].

[15] B.-H. Lee, C.-k. Lee and H. Min, Supersymmetric Chern-Simons vortex systems and fermion zero modes, Phys. Rev. D 45 (1992) 4588 [INSPIRE]. 
[16] J. Jaeckel and A. Ringwald, The Low-Energy Frontier of Particle Physics, Ann. Rev. Nucl. Part. Sci. 60 (2010) 405 [arXiv:1002.0329] [InSPIRE].

[17] P. Arias, D. Cadamuro, M. Goodsell, J. Jaeckel, J. Redondo and A. Ringwald, WISPy Cold Dark Matter, JCAP 06 (2012) 013 [arXiv:1201.5902] [INSPIRE].

[18] P. Arias, E. Ireson, F.A. Schaposnik and G. Tallarita, Chern-Simons-Higgs theory with visible and hidden sectors and its $N=2$ SUSY extension, Phys. Lett. B 749 (2015) 368 [arXiv: 1505.06705] [INSPIRE].

[19] M.M. Anber, Y. Burnier, E. Sabancilar and M. Shaposhnikov, Confined Vortices in Topologically Massive U(1) $\times \mathrm{U}(1)$ Theory, Phys. Rev. D 92 (2015) 065013 [arXiv: 1507.02709] [INSPIRE].

[20] M.M. Anber, Y. Burnier, E. Sabancilar and M. Shaposhnikov, Superconductivity at Any Temperature, arXiv:1508.05283 [INSPIRE].

[21] M.M. Anber, Y. Burnier, E. Sabancilar and M. Shaposhnikov, Diamagnetic Vortices in Chern Simons Theory, arXiv:1508.05284 [INSPIRE].

[22] C.-k. Lee, K.-M. Lee and E.J. Weinberg, Supersymmetry and Selfdual Chern-Simons Systems, Phys. Lett. B 243 (1990) 105 [INSPIRE]. 